\title{
ARTICLE
}

\section{A New Simulation Method of Geodesic Acoustic Mode in Toroidal Plasmas by Using Band-Limited White Noise in a $\delta f$ Neoclassical Transport Code}

\author{
Shinsuke SATAKE ${ }^{1,2, *}$, Hideo SUGAMA ${ }^{1,2}$, Ryutaro KANNO ${ }^{1,2}$, Takeshi IDO ${ }^{1,3}$, \\ Seikichi MATSUOKA ${ }^{2}$ and Masayuki YOKOYAMA ${ }^{1,2}$ \\ ${ }^{1}$ National Institute for Fusion Science, Oroshi 322-6, Toki, Gifu 509-5292, Japan \\ ${ }^{2}$ The Graduate University for Advanced Studies (Sokendai), Oroshi 322-6, Toki, Gifu 509-5292, Japan \\ ${ }^{3}$ Department of Energy Engineering and Science, Nagoya University, Nagoya 464-8603, Japan
}

\begin{abstract}
FORTEC-3D code, which solves the drift-kinetic equation for torus plasmas and radial electric field using the $\delta f$ Monte Carlo method, has developed to study the variety of issues relating to neoclassical transport phenomena in magnetic confinement plasmas. Here the numerical techniques used in FORTEC-3D are briefly reviewed, and recent progress in the simulation method to simulate GAM oscillation is also explained. A band-limited white noise term is introduced in the equation of time evolution of radial electric field to excite GAM oscillation, which enables us to analyze GAM frequency with fine resolution even in the case the collisionless GAM damping is fast.
\end{abstract}

KEYWORDS: Monte Carlo, neoclassical transport, pseudo-random number generator, white noise, FokkerPlanck-Landau collision operator, GAM oscillation

\section{Introduction}

Neoclassical transport theory ${ }^{1,2)}$ treats transport phenomena of plasmas in toroidal magnetic field configurations, which are governed by guiding-center motion and Coulomb collisions of charged particles. In torus plasmas, particles of which velocity pitch $\left|v_{\|} / v\right|$ is small are trapped in a region where magnetic field strength is weak, and those trapped particles mainly contribute the radial particle and heat transport. The plasma distribution function tends to deviate from local Maxwellian $f_{M}$ as the magnetic field is non-uniform and as the plasma collisionality is low. Then precise information of plasma distribution in the phase space is required to evaluate the transport. In neoclassical transport theory, the time evolution of plasma distribution function is described by the driftkinetic equation (DKE), which describes time evolution of gyro-averaged distribution function in five-dimensional phase space $\left(\mathbf{x}, v_{\|}, v_{\perp}\right)$.

From the early researches, DKE has been solved analytically or by computers adopting several approximations and simplifications, some of which are not always proper to simulate realistic cases in high-performance burning plasmas in forthcoming ITER experiments, ${ }^{3)}$ for example. Recent progress in computation environment enables us to solve DKE directly and to evaluate transport coefficients more correctly. We have developed FORTEC-3D code ${ }^{4-7)}$ which is applicable to general 3-dimensional magnetic field like helical configurations of Large Helical Device (LHD) in NIFS. ${ }^{8)}$ It treats self-consistent evolution of radial electric field and the finiteorbit-width (FOW) effect of guiding-center motion, which are usually neglected in conventional methods. FORTEC-3D has

*Corresponding author, E-mail:satake@nifs.ac.jp

(C) 2011 Atomic Energy Society of Japan, All Rights Reserved. been utilized to calculate transport coefficients in LHD plasmas with including radial electric field, and it is found that the non-local effect alters the equilibrium electric field profile. Its wide applicability is also being demonstrated in researches of recently concerned topics such as the geodesic acoustic mode (GAM) of electric field oscillation and Landau damping of it, ${ }^{5,7)}$ and simulation study of neoclassical toroidal viscosity in tokamaks with weak perturbation magnetic field. ${ }^{9)}$

In this paper, first we briefly review the numerical method of the $\delta f$ Monte Carlo method, which solves the DKE for deviation of distribution function from local Maxwellian, $\delta f=$ $f-f_{M}$. The conserved-form Monte Carlo collision operator and implementation of a parallelized pseudo-random numbers generator (PRNG) used in it are also explained. Then recent progress in the simulation method is shown about the excitation of GAM by introducing a band-limited white noise in the time evolution of the radial electric field. It enables us to analyze GAM frequency spectrum in LHD plasma, of which GAM damping is strong, by carrying out a long-duration GAM oscillation simulation with the excitation scheme.

\section{The $\delta f$ Monte Carlo Method}

FORTEC-3D solves the drift-kinetic equation for perturbed distribution function $\delta f=f-f_{M}$,

$$
\begin{aligned}
\frac{D}{D t} \delta f & \equiv \frac{\partial}{\partial t} \delta f+\left(\mathbf{v}_{\|}+\mathbf{v}_{d}\right) \cdot \nabla \delta f+\dot{\mathcal{K}} \frac{\partial \delta f}{\partial \mathcal{K}}-C_{T}(\delta f) \\
& =-\left(\mathbf{v}_{d} \cdot \nabla+\dot{\mathcal{K}} \frac{\partial}{\partial \mathcal{K}}\right) f_{M}+\mathcal{P} f_{M}
\end{aligned}
$$

where $f_{M}$ is a time-independent local Maxwellian which has constant density $n$ and temperature $T$ on a flux surface, $\mathbf{v}_{d} \equiv$ 
$\dot{\mathbf{x}}-v_{\|} \mathbf{b}$ is the drift velocity, $\mathbf{b}$ is the unit vector along the magnetic field line, and the independent velocity variables are chosen here as $\mathcal{K}=m v^{2} / 2$ and $\mu=m v_{\perp}^{2} /(2 B)$. FORTEC$3 \mathrm{D}$ adopts the Boozer magnetic coordinates ${ }^{10)}(\psi, \theta, \zeta)$, which is the toroidal magnetic flux within a flux surface, poloidal angle, and toroidal angle, respectively. The effective minor radius $r=a \sqrt{\psi / \psi_{\text {edge }}}$ is also used as a flux-surface variable, where $a=\sqrt{2 \psi_{\text {edge }} / B_{0}}, \psi_{\text {edge }}$ is the value of $\psi$ at the outermost flux surface, and $B_{0}$ is the magnetic field strength at the magntic axis $(r=0)$. Magnetic field in Boozer coordinates is given by

$\mathbf{B}=\nabla \psi \times \nabla \theta+\iota \nabla \zeta \times \nabla \psi=G \nabla \zeta+I \nabla \theta+\beta_{*} \nabla \psi$,

where $G(\psi)$ and $I(\psi)$ are the poloidal and toroidal current, and $\iota(\psi)$ is the rotational transform of the field line, respectively. The Jacobian of Boozer coordinates is $\sqrt{g_{B}}=(G+$ $\iota I) / B^{2}$. We assume that only electrostatic radial electric field $\mathbf{E}=-(d \Phi / d \psi) \nabla \psi=E_{\psi} \nabla \psi$ exists and the magnetic field is steady in time. Note that $\Phi$ is allowed to be time-dependent.

To solve Eq. (1), FORTEC-3D adopts the two-weight scheme of $\delta f$ Monte Carlo method. ${ }^{11,12)}$ Each simulation maker, of which distribution function is written as $g\left(\mathbf{x}, v_{\|}, v_{\perp}\right)$, has two weights which are defined so as to satisfy the relations $w g=\delta f$ and $p g=f_{M}$, respectively. Since each marker moves according to the 1.h.s. of Eq. (1), or $D g / D t=0$, time evolution of marker weights are given by

$$
\begin{aligned}
\dot{w} & =-\frac{p}{f_{M}}\left[\mathbf{v}_{d} \cdot \nabla+\dot{\mathcal{K}} \frac{\partial}{\partial \mathcal{K}}-\mathcal{P}\right] f_{M}, \\
\dot{p} & =\frac{p}{f_{M}}\left[\mathbf{v}_{d} \cdot \nabla+\dot{\mathcal{K}} \frac{\partial}{\partial \mathcal{K}}\right] f_{M} .
\end{aligned}
$$

The linearized Fokker-Planck-Landau collision operator (for like-particle collisions) is decomposed into the testparticle operator $C_{T}$ and the field-particle operator $\mathcal{P} f_{M}$. The former is implemented in a Monte Carlo way by a random walk of simulation marker velocity in the $\left(v_{\|}, v_{\perp}\right)$ space. $^{13)}$ The latter is defined so that the collision operator satisfies the following relations

$$
\begin{gathered}
\int d^{3} v \mathcal{M}\left(C_{T}(f)+\mathcal{P} f_{M}\right)=0 \\
\text { for } \mathcal{M}=\left\{1, v_{\|}, v^{2}\right\} \\
C_{T}(f)+\mathcal{P} f_{M}=0 \text { for } \delta f=\left(c_{0}+c_{1} v_{\|}+c_{2} v^{2}\right) f_{M}, \\
c_{i} \in R
\end{gathered}
$$

which describes the conservation property and the null-space of the linearized operator, respectively. The accuracy of the conservation property has improved recently by introducing new numerical scheme for $\mathcal{P} f_{M}$ operator. $\left.{ }^{6}\right)$

The time evolution of the radial electric field on each flux surface is given from the time derivative of Poisson's equation in Boozer coordinates as follows:

$$
\epsilon_{0}\left\langle|\nabla \psi|^{2}\left(1+\frac{c^{2}}{v_{A}^{2}}\right)\right\rangle_{\psi} \frac{\partial}{\partial t} E_{\psi}(\psi, t)=-\sum_{a} e_{a} \Gamma_{a}^{D},
$$

where $\langle\cdots\rangle$ denotes a flux-surface average defined as

$$
\langle A\rangle_{\psi} \equiv \frac{\oint d \theta d \zeta \sqrt{g_{B}} A(\psi, \theta, \zeta)}{\oint d \theta d \zeta \sqrt{g_{B}}},
$$

$v_{A}=\epsilon_{0} c B /\left(m_{i} n_{i}\right)^{1 / 2}$ is the Alfvén velocity, and the subscript $a$ specifies the particle species. The radial particle and heat fluxes are evaluated from the guiding-center radial drift velocity as follows:

$$
\begin{aligned}
\Gamma_{a}^{D}(\psi, t) & =\left\langle\int d^{3} v \delta f_{a} \mathbf{v}_{d} \cdot \nabla \psi\right\rangle_{\psi} . \\
Q_{a}^{D}(\psi, t) & =\left\langle\int d^{3} v \frac{m_{a}}{2} v^{2} \delta f_{a} \mathbf{v}_{d} \cdot \nabla \psi\right\rangle_{\psi} .
\end{aligned}
$$

In neoclassical transport simulations for LHD plasmas, determination of the ambipolar electric field profile, which evolves so that $\sum_{a} e_{a} \Gamma_{a}^{D}=0$ is satisfied on each flux surface, is one of the main tasks since $\Gamma_{a}^{D}$ and $Q_{a}^{D}$ in non-axisymmetric plasmas are strongly affected by $\mathbf{E} \times \mathbf{B}$ rotation speed. In order to reduce the computation time, FORTEC-3D only solves ion neoclassical transport, and another code GSRAKE ${ }^{14)}$ is used to evaluate $\Gamma_{e}^{D}$, which solves a bounce-averaged approximated DKE for helical configurations. In solving DKE for ions, FORTEC-3D neglects the ion-electron collisions because $m_{e} / m_{i} \ll 1$.

We have applied this method so far, because the finiteorbit-width effect and the strict conservation property of likeparticle collision term, which are the merit of FORTEC-3D, are considered to be more important for ion transport analysis than that for electron, because of the large mass difference. In the recent LHD experiments, however, core electron temperature becomes very high in Core Electron-Root Confinement (CERC) plasmas, ${ }^{15)}$ and positive ambipolar radial electric field is considered to play an important role to achieve good electron confinement. Since the $\mathrm{T}_{e}$ gradient in CERC is very steep and trapped electrons in such high- $\mathrm{T}_{e}$ plasmas is insensitive to Coulomb collisions and can drift widely in radial direction, it is concerned that the FOW effect on electron neoclassical transport in CERC plasmas would not be negligible. To simulate neoclassical transport and ambipolar electric field in CERC plasmas, FORTEC-3D is being extended for electron transport simulations. For that purpose, the unlikeparticle (electron-ion) collisions operator as well as the likeparticle ones is included. From benchmarks of the code, ${ }^{16)}$ it is found that the radial electron particle and heat fluxes in high- $\mathrm{T}_{e}$ LHD plasma are largely different from those evaluated from conventional neoclassical calculations especially if the $\mathbf{E} \times \mathbf{B}$ rotation is weak, which suggests the importance of precise calculation method of neoclassical transport in analyzing high- $T_{e}$ core plasmas.

FORTEC-3D is written in Fortran90 and parallelized by using MPI. In the parallelization of a massive Monte Carlo code, pseudo-random number generator (PRNG) may be a bottle neck. In a typical simulation run, FORTEC-3D uses about $1 \sim 4 \times 10^{7}$ markers and calculates about $10^{4} \sim 10^{5}$ times collisions, which requires a fast, long period, and statistically good quality PRNG. We have adopted a parallel PRNG according to Mersenne twister ${ }^{17)}$ with the dynamic creation method, ${ }^{18)}$ which generates independent random number sequences on each MPI process. FORTEC-3D is usually executed using 64 to 256 MPI processes with 8 parallel threads on shared memory, and the time cost of PRNG is less than $1 \%$ of the total simulation time. The parallel PRNG 
has passed a statistical independency test. ${ }^{19)}$ The Mersenne twister in FORTEC-3D can manage up to 1,024 MPI parallel computation now, though increase of communication time of $M P I$ REDUCE-type operations which are required in solving $\mathcal{P} f_{M}$ operator and Eq. (6) becomes bottle neck as the number of MPI processes increases.

\section{Excitation of GAM Using White-Noise}

Zonal flows and the geodesic acoustic mode (GAM) oscillation in toroidal plasmas have been investigated recently, as the zonal flow suppresses micro instabilities and anomalous transport. ${ }^{20)}$ Cross-device investigation ${ }^{21)}$ has revealed both similarities and differences in the nature of GAMs among several devices. One possibility to explain it is the magnetic field structure. Theoretical studies on GAMs in non-axisymmetric magnetic field based on the gyrokinetic model by Sugama and Watanabe $^{22)}$ have shown that the GAM frequency and damping rate depend on the magnetic field spectrum, and the collisionless damping of GAM oscillation in helical plasma is faster than that in a comparable scale tokamaks. To verify the analytic expectation of GAM in LHD magnetic configurations, we have carried out a simulation study of the dependence of GAM frequency and damping rate using FORTEC3D. ${ }^{5)}$ It has been shown there that GAM frequency $\omega_{G}$ is lower and the collisionless damping rate of GAM $\gamma_{G}$ is higher when the magnetic axis position of LHD is shifted inward. We have also shown there that the DKE simulation of FORTEC$3 \mathrm{D}$ corresponds to the $T_{e} / T_{i} \rightarrow 0$ limit of the gyrokinetic theory. Since executing global simulations of GAM in realistic configurations in a gyrokinetic model is time-consuming, researching GAM using a DKE model is considered as a useful tool. The reliability of FORTEC-3D for GAM simulation has been proved by a benchmark ${ }^{7)}$ with full- $f$ gyrokinetic Vlasov code GT5D. ${ }^{23)}$

In the previous study, however, we have used MHD equilibrium magnetic field of LHD with the rotational transform profile $\iota$ is fixed to a low value in surveying the GAM dependence on the magnetic axis position, $\iota=0.35$ to 0.5 at $r=0 \sim 0.8$, since the GAM damping rate is roughly proportional to $\iota$, and strong Landau damping occurs when $\iota \simeq 1$. In LHD experiments the $\iota$ profile is determined by the internal plasma current profile and magnetic axis position, usually increasing with minor radius. However, even if $\iota \ll 1$, the helical magnetic perturbations in LHD also causes rapid damping of GAM. Because of the strong GAM damping, it is difficult to analyze the frequency spectrum precisely from simulation result, since in evaluating GAM frequency by power-spectrum analyze, long-duration data of $\Gamma^{D}(\psi, t)$ and $E_{\psi}(\psi, t)$ are required to obtain a fine resolution in frequency. In the DKE model, however, there is basically no driving force of GAM, and the initial GAM in the simulation is excited just because the initial condition $\delta f=E_{\psi}=0$ is not an equilibrium state of toroidal plasma.

To resolve this problem, we devised a new technique of exciting GAM in DKE simulation. A white-noise term is introduced in the equation of time evolution of $E_{r}$ on a flux surface, which is described here as a stochastic differential equation as follows:

$$
\begin{gathered}
d E_{r}(r, t)=-\frac{e}{\epsilon_{0} \epsilon_{\perp}}\left(Z_{i} \Gamma_{i}^{D}(r, t)-\Gamma_{e}^{D}(r, t)\right) d t \\
+\sigma_{E}(r) d W(r, t),
\end{gathered}
$$

where the radial coordinate is transformed from $\psi$ to $r$ for simplicity, and the "diffusion coefficient" of radial electric field $\sigma_{E}$ is considered as a very small factor. Equation (7) can be regarded as a random-walk of radial electric field around an ambipolar state which mimics the effect of any turbulence on electrostatic potential in plasma. Concerning the noise term, a band-limited white-noise, of which frequency range contains the GAM frequency $\omega_{l}<\omega_{G}<\omega_{u}$, is adopted :

$$
d W(r, t)=\sqrt{\frac{2 d t}{N}} \sum_{k=1}^{N} \cos \left[\omega_{k} t+\phi_{k}(r)\right],
$$

where $N$ is sufficiently large number $(\gg 100), \omega_{k}=\omega_{l}+(k-$ $1 / 2) \Delta \omega, k=\{1,2, \cdots, N\}, \Delta \omega=\omega_{u}-\omega_{l}$, and the phase $\phi_{k}(r)$ are random numbers in $[0,2 \pi)$ given independently on each radial simulation mesh. Since the ambipolar electric field is $\mathcal{O}\left(T_{i} /(e a)\right)$ and $\omega_{G} \sim v_{t i} / R_{0}$ where $v_{t i}$ is ion thermal velocity and $R_{0}$ is the major radius, the diffusion coefficient $\sigma_{E}$ is given by

$$
\sigma_{E}^{2}(r)=\delta^{2}\left(\frac{T_{i}(r)}{e a}\right)^{2} \frac{v_{t i}(r)}{R_{0}},
$$

with a control parameter $\delta \ll 1$. Band-limited white noise is used so that GAM oscillation can be excited efficiently by small noise amplitude without bringing very high and low frequency components which are irrelevant to GAM excitation into the simulation. The idea of application of white noise is borrowed from the numerical method used in the researches of seismic resistant and structural analyses of buildings. ${ }^{24)}$ Since white-noise consists of continuum spectra, one can simulate the response of a structure to general earthquakes, without knowing details of the spectrum of "real" earthquake wave nor the eigenfrequncies of the structure. Similarly, though the physical driving mechanism of zonal flows and GAMs is regarded as nonlinear couplings of micro instabilities, which is beyond the drift-kinetic approach, we can still study the behavior of excited GAM with DKE by using white-noise instead of unknown driving term.

Numerically, Eq. (7) is solved by the Euler method, while marker motion and weight evolution are solved by the 4-th order Runge-Kutta method. To suppress the discretization error in time evolution, the time step size $d t$ is chosen small enough, $\omega_{G} d t, \omega_{u} d t \ll 10^{-2}$. We have checked the simulation results (frequency and amplitude of excited GAM) are not affected by the choice of $d t$ and $N$ in Eq. (8) by varying these parameters.

To demonstrate the numerical scheme, we carried out several simulations in a LHD magnetic field configuration $\left(R_{0}=\right.$ $3.75 \mathrm{~m}, B_{0}=1.5 \mathrm{~T}$, without plasma current) obtained from a MHD equilibrium calculation code VMEC. ${ }^{25)}$ FORTEC-3D adopts a Fourier series expression of magnetic field spectra as shown in Ref. 5). We used 22 components of $B_{m, n}$ spectra in the present study. Core ion density and temperature are $n_{i 0}=2.0 \times 10^{18} \mathrm{~m}^{-3}$ and $T_{i 0}=1.0 \mathrm{keV}$, respectively. 


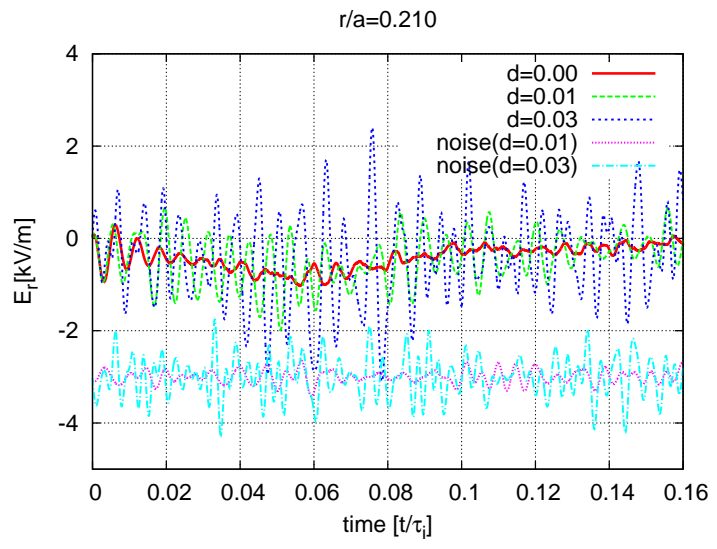

Fig. 1 Time evolution of radial electric field $E_{r}$ in GAM excitation simulations observed at $r / a=0.21$ surface. The time is normalized by the ion collision time $\tau_{i}$. The amplitude of the white-noise $\delta$ is varied $0.00,0.01$, and 0.03 in these simulations. The noise parts are plotted at the offcenter position in the graph with magnified by 200 .

The $\iota$ profile is monotonically increasing, $\iota(r=0)=0.34$, $\iota(0.56 a)=0.50$, and $\iota(a)=1.2$. The collisionality with these parameters was very low, and then the effect of collisions on GAM damping is negligible. The plasma configuration is made similar to a LHD experiment with observation of GAM. ${ }^{26,27)}$

The time evolution of the radial electric field and particle flux in the simulations are shown in Figs. 1 and 2, where three cases ( $\delta=0.00$ (without noise), 0.01 , and 0.03 ) are plotted. In Fig. 1, the given noise portions in $\delta=0.01$ and 0.03 cases are also plotted. Note that the noise amplitude is very small compared to the total $E_{r}$, then they are magnified by 200 in the figure. The GAM frequency $\omega_{G}$ is approximately estimated as

$$
\omega_{G 0}=\sqrt{7 / 4}\left(v_{t i} / R_{0}\right)\left(1+46 / 49 q^{2}\right)^{1 / 2}
$$

in the $T_{e} / T_{i} \rightarrow 0$ limit of simple axisymmetric tokamak configuration. In the present cases, it ranges about $26 \mathrm{kHz}$ $(r=0)>\omega_{G 0} /(2 \pi)>17 \mathrm{kHz}(r=a)$. Therefore, the band-limited white noise was given in the range from $\omega_{l} /(2 \pi)=10 \mathrm{kHz}$ to $\omega_{u} /(2 \pi)=36 \mathrm{kHz}$. In Figs. 1 and 2 , one can see that GAM was successfully excited continuously in time with very small amplitude of white noise. Comparing the amplitude of the noise part $\tilde{E}_{r}$ and the total $E_{r}$, the ratio is about $\left|\tilde{E}_{r} / E_{r}\right| \sim 1 / 100$, and excited GAM amplitude is roughly proportional to $\sigma_{E}$. On the other hand, if the white noise was turned off, initial GAM oscillation disappeared soon because of the strong Landau damping of GAM in helical configurations, and only a small fluctuation from Monte Carlo noise remained.

From the data of continuous wave form of GAM in particle flux $\Gamma_{i}^{D}(r, t)$ and radial electric field $E_{r}(r, t)$, we can analyze the GAM frequency by taking the power spectrum of them as it is shown in Figs. 3(a), (b) and (c). For $\delta=0.01$ case, the peak of the spectrum appears slightly below the estimation $\omega_{G 0}$, which is an approximation by neglecting the helicity of

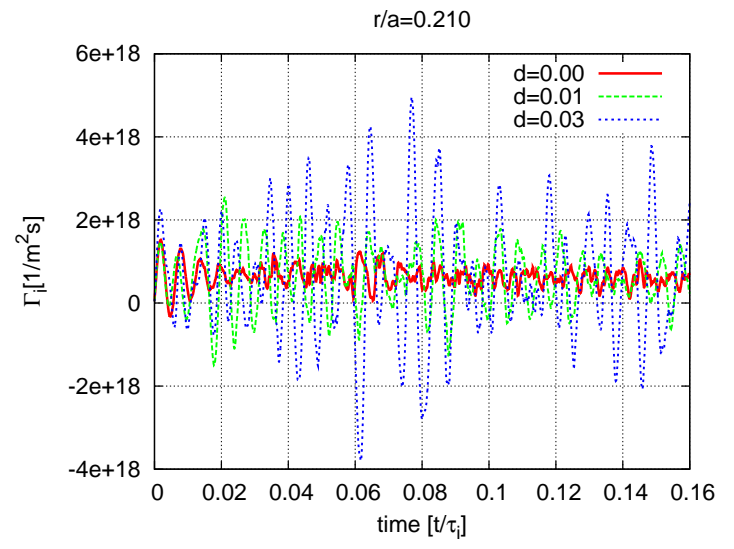

Fig. 2 Time evolution of radial particle flux $\Gamma_{i}$ in GAM excitation simulations observed at $r / a=0.21$ surface. The amplitude of the white-noise $\delta$ is varied $0.00,0.01$, and 0.03 in these simulations.

LHD magnetic field. The band-limited white noise has uniform power spectrum in the range from $\omega_{l}$ to $\omega_{u}$ as shwon in Fig. 3(c). On the other hand, we cannot see clear peak of power spectrum if the white noise is cut as in Fig. 3(a). Though we do not show the power spectrum of $\Gamma_{i}^{D}$, it has almost the same profile as that of $E_{r}$. The radial position where clear peak of GAM oscillation is $r / a=0.05 \sim 0.20$, which seems consistent with the observation of GAM using HeavyIon-Beam-Probe (HIBP) in a LHD experiment, ${ }^{27}$ ) of which profile is similar to the one in the simulation. We have also confirmed that the GAM frequency and the peak radial position of GAM in simulation are almost unaffected by varying the white-noise amplitude from $\delta=0.01$ to 0.03 .

Next, the peak frequencies and peak amplitudes of the power spectrum of $\Gamma_{i}^{D}$ and $E_{r}$ are compared with the gyrokinetic theory, ${ }^{22)}$ which takes into account of the helical magnetic ripples of LHD configurations. Analytic solutions of $\omega_{G}$ and $\gamma_{G}$ for the gyrokinetic analysis in the $T_{e} / T_{i} \rightarrow 0$ drift-kinetic limit is shown in Eqs. (7) and (8) in Ref. 5). Figure 4(a) shows the radial profile of GAM frequency. Note that $\omega_{G 0}$ is an approximate estimation of GAM frequency in a tokamak geometry. Both gyrokinetic analysis and FORTEC$3 \mathrm{D}$ simulation show lower $\omega_{G}$ than $\omega_{G 0}$, which is the effect of helical configuration of LHD magnetic field. The radial profile of the peak of power spectrum amplitudes of $\Gamma_{i}^{D}$ and $E_{r}$ are plotted in Fig. 4(b). The peak of amplitude is located at $r / a \simeq 0.125$, and the amplitudes decay at outboard region. It was diffucult to find a clear peak in power spectrum and identify $\omega_{G}$ from simulation results at $r / a>0.35$. It is because both $\iota$ and the helical ripple components of magnetic field spectrum are increasing function of $r$ in the LHD configuration used here, which results in the larger damping rate of GAM, $\left|\gamma_{G}\right|$. One can see that the peak amplitudes observed in FORTEC-3D simulation tend to be proportional to $\left|1 / \gamma_{G}\right|$, which means that the GAM excited by the white noise actually damps according to the kinetic mechanism that gyrokinetic theory explains. Recent analysis of LHD experiment has found that the spacial profile of GAM power shows the sim- 
(a) Power Spectrum $E_{r}: d=0.00$

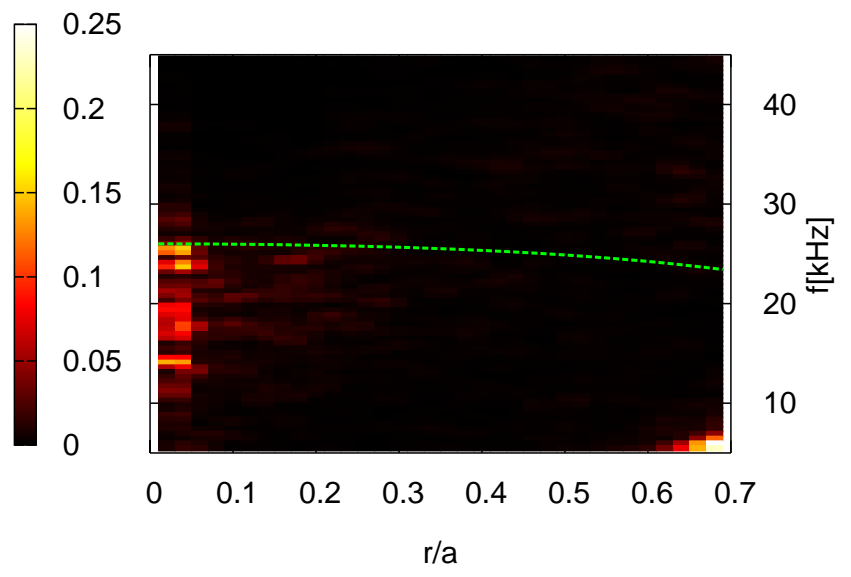

(b) Power Spectrum $E_{r}: d=0.01$

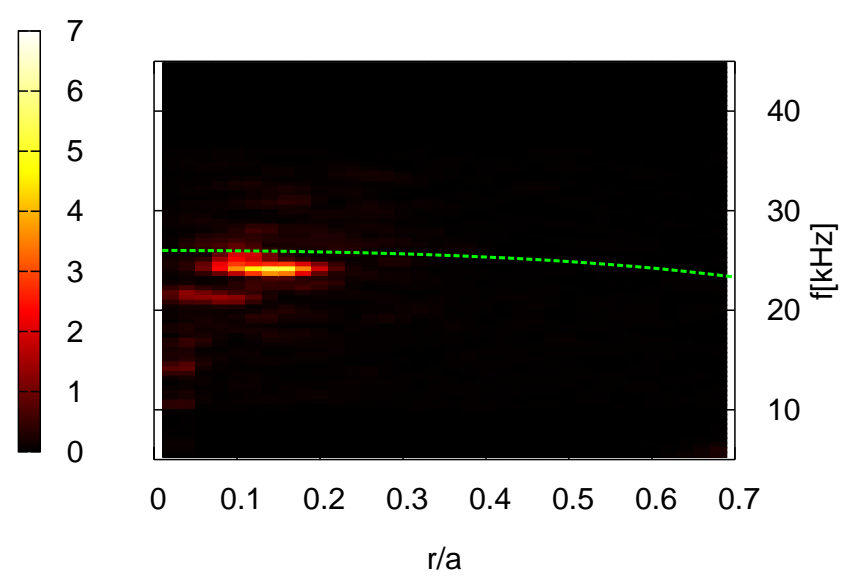

(c) White noise : $d=0.01$

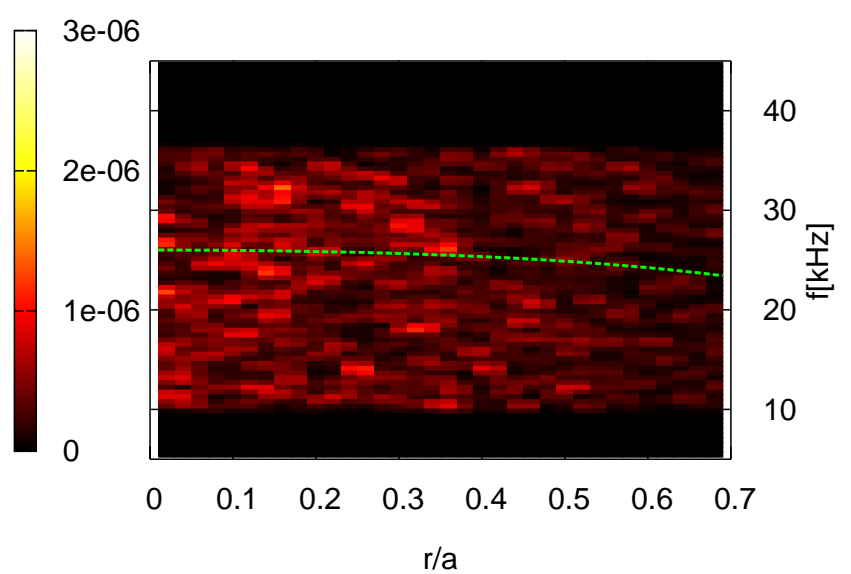

Fig. 3 Contour plots of the power spectrum of $E_{r}$ for (a): $\delta=0.00$ and (b): $\delta=0.01$ case, respectively, and (c): the power spectrum of the white-noise part for $\delta=0.01$ case. Green line represents an approximate estimation of GAM frequency for tokamak case, Eq. (10). Note that the scale of the color contour is different among these figures. ilar tendency as Fig. 4(b). ${ }^{27)}$ This is simply explained using a model of forced vibration of an oscillator of which natural frequency is $\omega_{0}$,

$$
m \ddot{x}+\gamma \omega_{0} \dot{x}+\omega_{0}^{2} x=f(t),
$$

where $\gamma$ is the damping rate. If the driving force $f(t)$ is whitenoise of which power spectral density is $S(\omega)=S_{0}$, the variation of the displacement $\sigma_{x}^{2}=E\left[x^{2}(t)\right]$ is known to be proportional to $S_{0} /\left(\gamma \omega_{0}^{3}\right),{ }^{24)}$ where $E[g]$ means the expected value of $g$. Note here that $\sigma_{E}^{2} \propto S_{0}$ in Eq. (7). Though Eq. (11) is a simple model of GAM damping by neglecting radial correlations, it explains that $E\left[\left|E_{r}-\overline{E_{r}}\right|^{2}\right] \propto\left|\gamma_{G}\right|^{-1}$. We could have seen GAM at $r / a>0.35$ if the driving term had been much stronger, however, the coincidence of the radial profile of the GAM power between simulations and observation implies that the realistic choice of the GAM driving term $\delta$ is the order of $10^{-2}$. More investigation by comparing the GAM oscillation amplitude between simulation and observation is planned near future.

Finally, at the closest position to the magnetic axis $r / a=$ 0.05 , difference between simulation and theory is large. It is possibly because of the FOW effect of passing particle orbit around the magnetic axis, of which radial drift width is wide there (so called potato orbits), or because of the effect of boundary condition $E_{r}(r=0)=0$ in the simulation. The gyrokinetic analysis do not assume the existence of potato orbits nor the magnetic axis.

\section{Summary}

We have developed a new simulation scheme to simulate GAM in LHD plasmas, in which the GAM damping rate is essentially high compared with that in tokamaks, by applying a band-limited white noise as a driving term of GAM oscillation in FORTEC-3D DKE Monte Carlo code. From the test simulations in a LHD magnetic configuration it has been demonstrated that the GAM oscillation can be excited by very small amplitude of white noise, and the GAM frequency and the peak amplitude of the power spectrum of GAM oscillation are consistent with a gyrokinetic analysis for helical plasmas. With the GAM excitation scheme FORTEC-3D simulation is able to simulate GAM oscillation and damping in realistic LHD plasma profile and analyze GAM power spectrum with fine resolution. LHD is equipped with HIBP which observes electrostatic potential profile in core plasma. GAM frequency profile can be obtained from the diagnostics, and then we can carry out precise comparisons among observation, gyrokinetic theory, and FORTEC-3D simulation, which will be profitable for further understanding the physics of GAMs and zonal flows in helical plasmas. Further improvement of the simulation model is planned in near future to include the impurity heavy ion effect, which is one of the candidates that change the observed GAM frequency from the analytic estimation.

\section{Acknowledgments}

This work is supported in part by the Japanese Ministry of Education, Culture, Sports, Science, and Technology, Grant No. 21560861 and in part by the NIFS collab- 
(a) Peak frequency

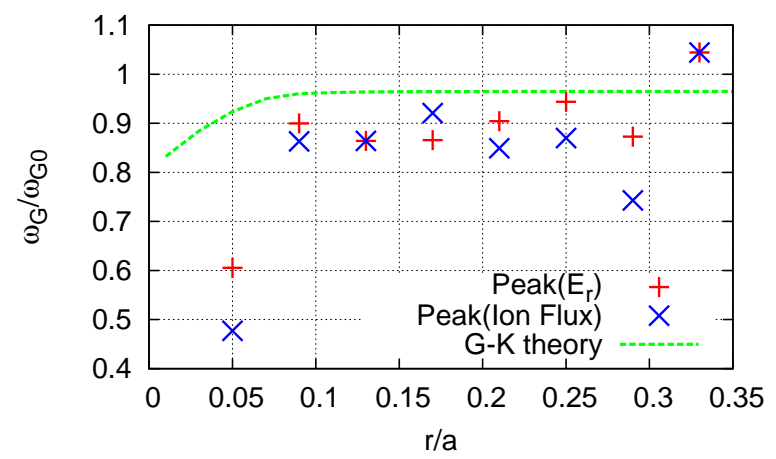

(b) Peak amplitude

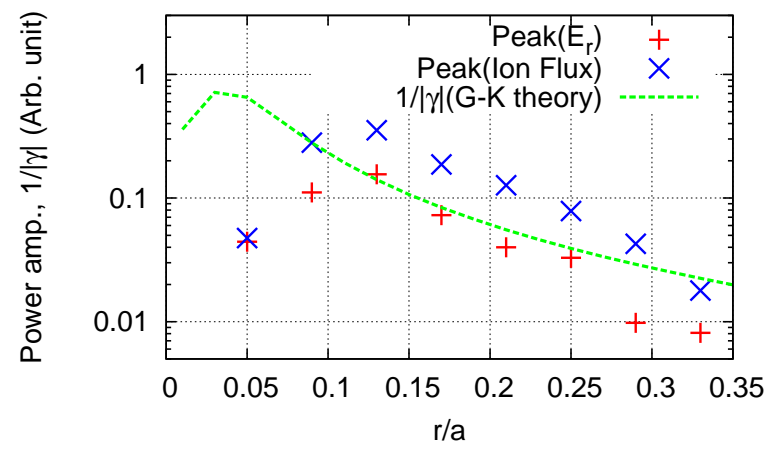

Fig. 4 (a): Comparisons of GAM frequency estimated from peak positions of power spectrum of $E_{r}$ and $\Gamma_{i}^{D}$ (symbols), and an estimation from gyrokinetic analysis ${ }^{5,22}$ (line). The vertical axis is normalized by an approximate estimation for tokamak case, Eq. (10). (b): Comparisons of the peak amplitudes of power spectrum of $E_{r}$ and $\Gamma_{i}^{D}$ (symbols), and the inverse of GAM damping rate $1 /\left|\gamma_{G}\right|$ from gyrokinetic analysis (line). FORTEC-3D results for $\delta=0.03$ case are used here.

orative Research Programs, NIFS09KTAD006, 09KTAL022, 10KDAT020, 10KNXN186, and 10KTAT040.

\section{References}

1) R. Balescu, Transport Processes in Plasmas Vol. 1 \& 2, NorthHolland, Amsterdam (1998).

2) P. Helander, D. J. Sigmar, Collisional Transport in Magnetized Plasmas, Cambridge Univ. Press, Cambridge (2002).

3) ITER Physics Basis Editors et al., Nucl. Fusion, 39, 2137 (1999).

4) S. Satake, M. Okamoto, N. Nakajima, H. Sugama, M. Yokoyama, C. D. Beidler, "Non-Local Simulation of the Formation of Neoclassical Ambipolar Electric Field in NonAxisymmetric Configurations," Plasma Fusion Res., 1, 002 (2006).

5) S. Satake, H. Sugama, T.-H. Watanabe, "Simulation studies on the GAM oscillation and damping in helical configurations," Nucl. Fusion, 47, 1258 (2007).

6) S. Satake, R. Kanno, H. Sugama "Development of a NonLocal Neoclassical Transport Code for Helical Configurations," Plasma Fusion Res., 3, S1062 (2008).

7) S. Satake, Y. Idomura, H. Sugama, T.-H. Watanabe, "Benchmark test of drift-kinetic and gyrokinetic codes through neoclassical transport simulations," Comput. Phys. Comm., 181, 1069 (2010).
8) A. Iiyoshi, A. Komori, A. Ejiri et al., "Overview of the Large Helical Device project," Nucl. Fusion, 39, 1245 (1999).

9) S. Satake, H. Sugama, R. Kanno, J.-K. Park, "Calculation of Neoclassical Toroidal Viscosity in Tokamaks with Broken Toroidal Symmetry," Plasma Phys. Contr. Fusion, 53, 054018 (2011).

10) A. H. Boozer, "Plasma equilibrium with rational magnetic surfaces," Phys. Fluids, 24, 1999 (1981).

11) W. X. Wang, N. Nakajima, M. Okamoto, S. Murakami, "A new $\delta f$ method for neoclassical transport studies," Plasma Phys. Contr. Fusion, 41, 1091 (1999).

12) S. Brunner, E. Valeo, A. Krommes "Collisional delta-f scheme with evolving background for transport time scale simulations," Phys. Plasmas, 6, 4504 (1999).

13) X. Q. Xu, M. N. Rosenbluth, "Numerical simulation of iontemperature-gradient-driven modes," Phys. Fluids, B3, 627 (1991).

14) C. D. Beidler, H. Maaßberg "An improved formulation of the ripple-averaged kinetic theory of neoclassical transport in stellarators," Plasma Phys. Contr. Fusion, 43, 1131 (2001).

15) M. Yokoyama, H. Maaßberg, C. D. Beidler et al., "Common Features of Core Electron-Root Confinement in Helical Devices," Fusion Sci. Technol., 50, 327 (2006).

16) S. Matsuoka, S. Satake, M. Yokoyama, A. Wakasa, S. Murakami, "Neoclassical electron transport calculation by using $\delta f$ Monte Carlo method," Phys. Plasmas, 18, 032511 (2011).

17) M. Matsumoto, T. Nishimura "Mersenne twister: a 623dimensionally equidistributed uniform pseudo-random number generator," ACM Trans. Model. Comput. Simulat., 8, 3 (1998).

18) M. Matsumoto, T. Nishimura, "Dynamic Creation of Pseudorandom Number Generators," Monte Carlo and Quasi-Monte Carlo Methods 1998, Springer, 56 (2000).

19) S. Satake, M. Okamoto, N. Nakajima, H. Takamaru, "Development of three-dimensional neoclassical transport simulation code with high performance Fortran on a vector-parallel computer," Lecture notes in Computer Science, 4759, SpringerVerlag, Berlin (2008).

20) K. Itoh, S.-I. Itoh, P. H. Diamond et al., "Physics of zonal flows," Phys. Plasmas, 13, 055502 (2006).

21) A. Fujisawa, T. Ido, A. Shimizu et al., "Experimental progress on zonal flow physics in toroidal plasmas," Nucl. Fusion, 47, S718 (2007).

22) H. Sugama, T.-H. Watanabe, "Collisionless damping of zonal flows in helical systems," Phys. Plasmas, 13, 012501 (2006).

23) Y. Idomura, M. Ida, T. Kano, N. Aiba and S. Tokuda, "Conservative global gyrokinetic toroidal full- $f$ five-dimensional Vlasov simulation," Comput. Phys. Comm., 179, 391 (2008).

24) M. Hoshiya, Kakuritsuron shuho ni yoru shindo kaiseki, Kajima Kenkyujo Shuppankai, Chapters 3 and 4 (1974), [in Japanese].

25) S. P. Hirshman, O. Betancourt, "Preconditioned descent algorithm for rapid calculations of magnetohydrodynamic equilibria," J. Comput. Phys., 96, 99 (1991).

26) T. Ido, A. Shimizu, M. Nishiura et al., "Experimental study of radial electric field and electrostatic potential fluctuation in the Large Helical Device," Plasma Phys. Contr. Fusion, 52, 124025 (2010).

27) T. Ido, A. Shimizu, M. Nishiura et al., "Potential fluctuation associated with the energetic-particle-induced geodesic acoustic mode in the Large Helical Device," Nucl. Fusion, 51, 073046 (2011). 